\title{
Neural substrate of concurrent sound perception: direct electrophysiological recordings from human auditory cortex
}

\author{
Aurélie Bidet-Caulet ${ }^{1,2,3, *}$, Catherine Fischer ${ }^{1,2,3,4}$, Françoise Bauchet ${ }^{1,2,3,5}$, \\ Pierre-Emmanuel Aguera ${ }^{1,2,3}$ and Olivier Bertrand ${ }^{1,2,3}$
}

1. INSERM, U821, Lyon, France

2. Institut Fédératif des Neurosciences, Lyon, France

3. Université de Lyon, Lyon, France

4. Functional Neurology and Epileptology Department, Neurological Hospital, Lyon, France

5. MEG Department, CERMEP, Lyon, France

Edited by: Robert T. Knight, University of California Berkeley, USA

Reviewed by: Gregory McCarthy, Yale University, USA

James P. Morris, Duke University Medical Centre, USA

\begin{abstract}
In everyday life, consciously or not, we are constantly disentangling the multiple auditory sources contributing to our acoustical environment. To better understand the neural mechanisms involved in concurrent sound processing, we manipulated sound onset asynchrony to induce the segregation or grouping of two concurrent sounds. Each sound consisted of amplitude-modulated tones at different carrier and modulation frequencies, allowing a cortical tagging of each sound. Electrophysiological recordings were carried out in epileptic patients with pharmacologically resistant partial epilepsy, implanted with depth electrodes in the temporal cortex. Patients were presented with the stimuli while they performed an auditory distracting task. We found that transient and steady-state evoked responses, and induced gamma oscillatory activities were enhanced in the case of onset synchrony. These effects were mainly located in the Heschl's gyrus for steady-state responses whereas they were found in the lateral superior temporal gyrus for evoked transient responses and induced gamma oscillations. They can be related to distinct neural mechanisms such as frequency selectivity and habituation. These results in the auditory cortex provide an anatomically refined description of the neurophysiological components which might be involved in the perception of concurrent sounds.
\end{abstract}

Keywords: auditory cortex, stream segregation, EEG, gamma, event-related, steady-state, human

\section{INTRODUCTION}

In ecological situations, we are often confronted with a mixture of sounds produced by several simultaneously active sources and it is crucial to be able to parse and identify these multiple sources. In fact, within this mixture, independently of the orientation of our attention, we can suddenly detect one acoustic sequence or stream that can be clearly isolated from other sounds and attributed it to a source, like a bird song in the forest or a motorbike acceleration in a noisy street. This acoustic segregation relies on the ability of our auditory system to group together or to dissociate acoustic components at a given instant and over time, leading to the perception of several simultaneous auditory streams.

Many psychoacoustical studies (Bregman, 1990) have shown the contribution of various acoustic features (e.g., pitch, timbre, location, onset synchrony) in automatically segregating or grouping acoustic events. In particular, the acoustical context in which a sound is presented can highly influence its perception, i.e., either as belonging to a pre-existing stream

*Correspondence: Aurélie Bidet-Caulet, INSERM, Lyon, F-69500, France; Institut Fédératif des Neurosciences, Lyon, F-69000, France; Université Claude Bernard Lyon 1, Lyon, F-69000, France. e-mail: bidet-caulet@lyon.inserm.fr

Received: 15 September 2007; paper pending published: 29 November 2007; accepted: 03 January 2008; published online: 28 March 2008.

Citation: Front. Hum. Neurosci. (2008) 1: 5. doi: 10.3389/neuro.09.005.2007

Copyright (C) 2008 Bidet-Caulet, Fischer, Bauchet, Aguera and Bertrand. This is an open-access article subject to an exclusive license agreement between the authors and the Frontiers Research Foundation, which permits unrestricted use, distribution, and reproduction in any medium, provided the original authors and source are credited. or constituting a new one. This has been confirmed by electrophysiological recordings in humans (Alain and Izenberg, 2003; Nager et al., 2003; Sussman et al., 1999, 2005; Takegata et al., 2005; Winkler et al., 2003). Nevertheless, few studies have investigated the neural mechanisms involved in the perceptual organization of sounds, and, among those, most of them have investigated the segregation of alternating sounds (see Micheyl et al., 2007 for a review). The present study is focusing on the neural mechanisms by which the auditory system dissociates or groups overlapping auditory components, as a function of the acoustical context. These neural mechanisms could be reflected in different electrophysiological responses such as evoked transient and sustained responses, steadystate responses (elicited by amplitude-modulated sounds) and induced gamma oscillations. Indeed, different evoked responses were found for harmonic (grouped percept) and mistuned (segregated percept) stimuli, in the chinchilla inferior colliculus (Sinex et al., 2002) and in human auditory cortex (Alain et al., 2001, 2002; Dyson and Alain, 2004). Moreover, the steady-state responses can be used for tagging the electrophysiological activity corresponding to each concurrent component independently, when they have distinct amplitude modulation frequencies (Bidet-Caulet et al., 2007; Draganova et al., 2002; Lins and Picton, 1995). Finally, induced gamma oscillatory activities $(20-100 \mathrm{~Hz})$ are assumed to be involved in the construction of coherent objects (Bertrand and Tallon-Baudry, 2000) and could thus play an important role in auditory grouping. It is thus hypothesized that these different electrophysiological responses could be differentially modulated according to the percept, in particular that gamma oscillations could be increased in the case of auditory grouping. 
In the present electrophysiological study, we manipulated sound onset asynchrony to induce two perceptual situations, the segregation or grouping of two concurrent sounds (Bregman, 1990; Darwin et al., 1995; Turgeon et al., 2002). Stimuli were made of two partially overlapping components having different carrier frequencies and amplitude modulation frequencies (21 and $29 \mathrm{~Hz}$ ): the $21-\mathrm{Hz}$ and $29-\mathrm{Hz}$ components (Figure 1). To have a similar acoustical structure across conditions, the 21- $\mathrm{Hz}$ component always started first (part 1), and then, at the onset of the 29-Hz component (part 2), its carrier frequency could be kept constant (pitch continuity) or shifted (pitch discontinuity). To reduce electrophysiological differences due to the acoustical properties of the sounds, stimuli were identical in the last part, in the two conditions. By behavioral testing, we assessed that, in case of pitch continuity of the $21-\mathrm{Hz}$ component (i.e., onset asynchrony), the two components were perceived as two distinct streams during part 2 (2-stream condition), whereas in case of pitch discontinuity (i.e., onset synchrony), they were perceived as one complex stream (1-stream condition). In other words, in the 2-stream condition, the second sound (29-Hz component) was perceived as an additional acoustic stream superimposed to and distinct from the first one (21- $\mathrm{Hz}$ component), while in the 1-stream condition, the second sound was merging to the first one to form a single and new complex stream. We used intracranial electrophysiological (EEG) recordings of epileptic patients with pharmacologically resistant epilepsy, implanted with multicontact depth electrodes in the temporal cortex. This constitutes a unique approach for providing a precise time-course and localization of the neurophysiological correlates of concurrent sound perception in humans. During the electrophysiological recordings, the patients were presented with both types of stimuli and performed an auditory distracting task which required focusing their attention away from the streams. Analyzing part 2 of the stimulus, when both components are present, allowed us to compare the electrophysiological responses to acoustically identical stimuli, corresponding to distinct percepts (one or two streams) because of different history in the part 1, i.e., different acoustical contexts. We investigated different electrophysiological components (transient and sustained evoked responses, steady-state responses and gamma oscillatory activities) to have a comprehensive insight into the neural mechanisms that could be involved at distinct processing levels, in different cortical auditory areas (Heschl's gyrus, planum temporale and polare). From the analysis of these complementary electrophysiological components, directly recorded from the human auditory cortex, we suggest that the implicit segregation or grouping of two concurrent sounds could rely on the interaction of several neurophysiological mechanisms, highly related to the acoustic context, occurring at different latencies and locations in the human auditory system.

\section{MATERIALS AND METHODS Electrophysiological experiment}

Patients. Five patients (4 females and 1 male, age ranging from 22 to 36 years) suffered from pharmacologically resistant partial epilepsy and were candidates for surgery. Because the location of the epileptic focus could not be identified using non-invasive methods, they were stereotactically implanted with multicontact depth probes. Electrophysiological recording is part of the brain functional evaluation that is performed routinely before epilepsy surgery in patients implanted with depth electrodes. According to the French regulations concerning invasive investigations with a direct individual benefit, patients were fully informed about the electrode implantation, stereotactic EEG and evoked potential recordings and the cortical stimulation procedures used to localize the epileptogenic and functional brain areas. All patients gave their informed consent to participate in the experiment. The signals described here were recorded away from the seizure focus. When the recording sessions took place, antiepileptic drugs administered to the patients had been either discontinued or drastically reduced for the purpose of recording seizures. No patient was administered benzodiazepines. None of the patients reported any auditory complaint.
Stimuli and task. Stimuli were composed of two sounds which could be perceived as 1 complex stream or 2 distinct streams, corresponding to the 1-stream or 2-stream condition, respectively. They were randomly presented to patients in 4 blocks of 80 trials (resulting in 160 repetitions in each condition). They had to perform a diverted attention task which consisted of the detection of rare noise bursts superimposed to the stimuli, thus orienting their attention away from the stimulus content. They were instructed to answer as soon as they heard a noise burst, by pressing a button. The task presented no major difficulty but was demanding enough to keep the patients alert: all the patients detected all the noise bursts, except one who missed two of them. After the recording session, patients reported that they did not pay attention to the stimuli on which the noise bursts were superimposed and did not notice the presence of two types of stimuli.

All stimuli were composed of two successive parts (Figure 1). Part 1 lasted 0.810 second (1 second for the first patient) and the duration of part 2 was equiprobably chosen between $0.810,0.905,1$ and 1.095 seconds $(1.095,1.190,1.286$ and 1.381 seconds for the first patient). During part 2, all stimuli are made of two components: the $21-\mathrm{Hz}$ component and the $29-\mathrm{Hz}$ component. The $21-\mathrm{Hz}$ component was composed of two amplitude-modulated tones separated by two octaves, the carrier frequency of the lower one being equiprobably chosen between $659,698,740$ and $784 \mathrm{~Hz}$. These two tones were both amplitude modulated in phase at a frequency of $21 \mathrm{~Hz}$. The $29-\mathrm{Hz}$ component consisted of one tone separated by one octave from each tone of the first sound. This tone was amplitude modulated at a frequency of $29 \mathrm{~Hz}$. Thus, during part 2, all stimuli were acoustically identical. To have a similar acoustical structure across stimulus conditions, the $21-\mathrm{Hz}$ component was always present during part 1. For 2-stream stimuli, the carrier frequency of the 21- $\mathrm{Hz}$ component was maintained constant (pitch continuity) whereas for 1 -stream stimuli it was shifted by \pm 3 semitones (pitch discontinuity). Therefore, in the case of 2-stream stimuli, the 21- $\mathrm{Hz}$ component started before the $29-\mathrm{Hz}$ component, which corresponded to an onset asynchrony and induced auditory segregation and the perception of two distinct streams. Conversely, in the case of 1-stream stimuli, the two acoustic components started at the same time (onset of part 2) inducing a grouping of the two sounds into one single complex stream.

The superimposed target sounds were 150 -ms band-pass filtered noise bursts ( 20 -semitone wide starting at $784 \mathrm{~Hz}$ with $10 \mathrm{~ms}$ rise/fall time). The targets were delivered in $15 \%$ of the stimuli and randomly occurred during the stimulus, $0.2,0.5,1.2$ or 1.5 seconds before the end of the stimulus. When a target was present in a trial, the next stimulus started between 0.7 and 1.0 second after the patient's response, otherwise the inter-trial interval was randomized between 0.9 and 1.4 seconds.

The intensities of all tones were corrected according to their carrier frequency (Botte et al., 1989, Figure 1.2, p.17) and then, 21- $\mathrm{Hz}$ and $29-\mathrm{Hz}$ components were matched in intensity. Stimuli were delivered at an intensity level judged comfortable by the patient at the beginning of the experiment (about $70 \mathrm{~dB} \mathrm{SPL}$ ). Noise bursts were $5 \mathrm{~dB}$ above the stimulus intensity level. All component onsets and offsets were linearly ramped during $10 \mathrm{~ms}$. In case of 1 -stream stimuli, the $21-\mathrm{Hz}$ component was also ramped on both sides of the pitch discontinuity.

Stimulus duration, carrier frequency and noise burst occurrence were randomized to limit habituation and predictability.

Recording and signal analysis. Intracranial recordings were performed at the Functional Neurology and Epilepsy Department (Lyon Neurological Hospital). EEG was recorded from 64 intracranial electrode contacts referenced to an intracranial contact away from the superior temporal cortex (ground electrode being at forehead). Signals were amplified, filtered (0.1-200 Hz bandwidth) and sampled at $1000 \mathrm{~Hz}$ (Synamps, Neuroscan Labs, Sterling, VA, USA).

The analysis was restricted to the electrodes located in the temporal cortex and its immediate vicinity. Raw data were visually inspected and trials showing epileptic spikes were discarded. Because of excessive epileptic spikes, patient \#3 was excluded from evoked response analysis but 


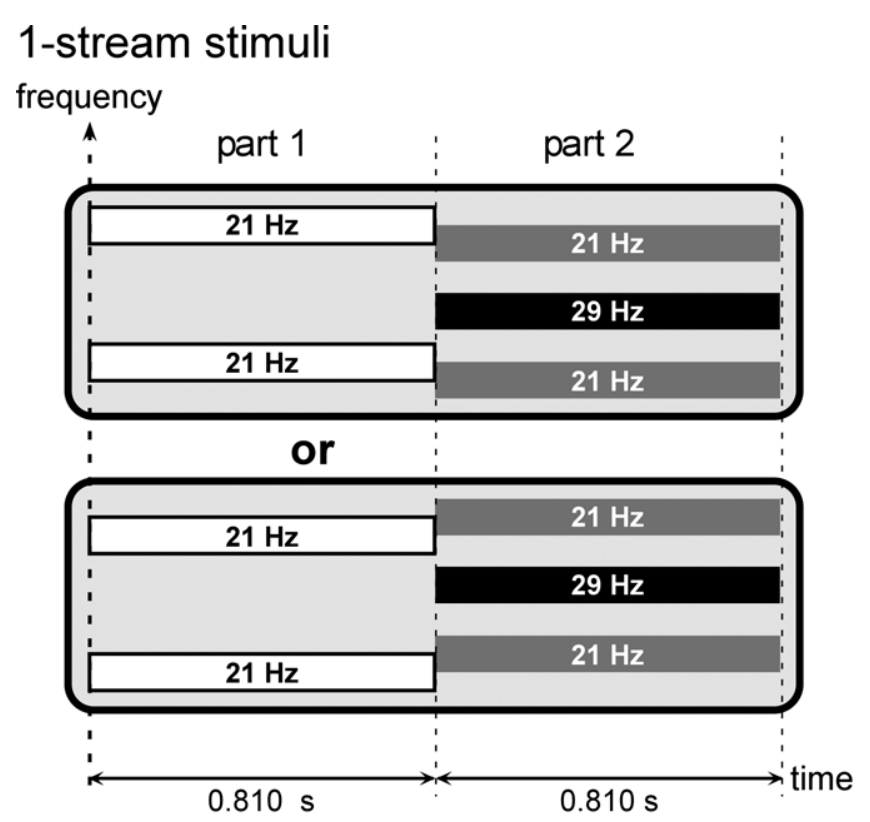

2-stream stimulus

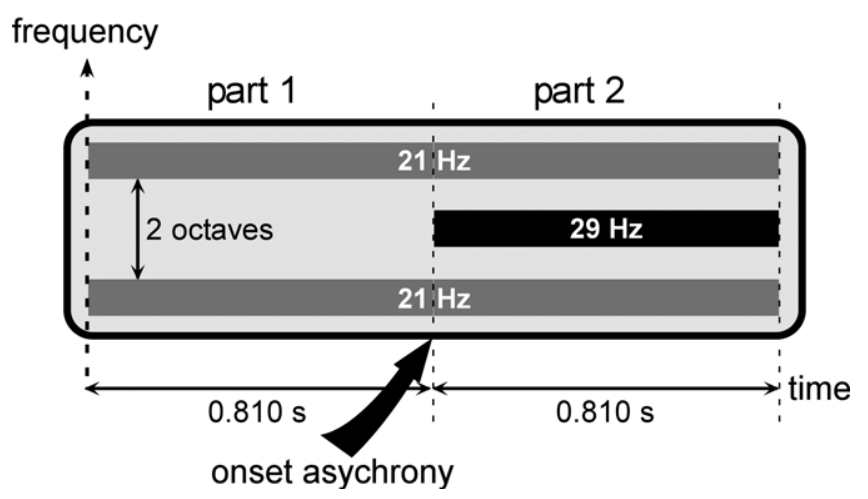

Figure 1. Stimuli. Stimuli were composed of two sounds which could be perceived as 1 complex stream or 2 distinct streams, corresponding to the 1-stream or 2-stream condition, respectively. All stimuli were composed of two parts lasting at least 0.810 second (see details in the text). During part 2 , all stimuli consisted of two components. The 21-Hz component (gray bars) was composed of two tones, separated by two octaves, and amplitude modulated at a frequency of $21 \mathrm{~Hz}$. The 29- $\mathrm{Hz}$ component (black bar) consisted of one tone, separated by one octave from each tone of the first component, and amplitude modulated at a frequency of $29 \mathrm{~Hz}$. During part 2, all stimuli were thus acoustically identical. To have a similar acoustical structure across stimulus conditions, the 21-Hz component was always present during part 1. For 2-stream stimuli, the carrier frequency of the 21-Hz component (gray bars) was maintained constant (pitch continuity) whereas for 1-stream stimuli the $21-\mathrm{Hz}$ component (white bars) was shifted by \pm 3 semitones (pitch discontinuity). Therefore, in the case of 2-stream stimuli, the 21- $\mathrm{Hz}$ component started before the 29-Hz component, which corresponded to an onset asynchrony and induced auditory segregation and the perception of two distinct streams. Conversely, in the case of 1-stream stimuli, the two acoustic components started at the same time (onset of part 2) inducing a grouping of the two sounds into one single complex stream.

kept for steady-state and gamma oscillation analysis because their frequency bands were less contaminated by the spectral content of epileptic spikes. Trials with a noise burst occurring before 1.620 seconds (about $12 \%$ of all trials) and incorrect trials were rejected from further analysis. The mean numbers of correct and non-artifacted trials were 134 and 131.6 out of 160 for 1-stream and 2-stream conditions, respectively.
ERPs were averaged during the shortest duration of each part (i.e. 0.810 second for each part), separately for the two conditions. The ERP of the two periods (parts) were corrected with respect to the same baseline defined between -150 and 0 ms before part 1 stimulus onset.

Both the steady-state periodic response, evoked by the amplitude modulation of the sound components at 21 and $29 \mathrm{~Hz}$ (phase-locked to the stimulus content) and the beta/gamma oscillations, in the $20-100 \mathrm{~Hz}$ range, induced by the stimulus (jittering in latency from trial to trial) were analyzed by means of a wavelet decomposition which provides a good compromise between time and frequency resolutions. Each singletrial signal was transformed in the time-frequency domain by convolution with complex Gaussian Morlet's wavelets with a ratio $f / \sigma_{f}$ of 10 with $f$ being the central frequency of the wavelet and $\sigma_{f}$ its standard deviation (Tallon-Baudry and Bertrand, 1999, for details). The resulting time-frequency powers were then averaged across trials. This method led to a power estimate of both evoked and induced activities in the time-frequency (TF) domain. A baseline correction was applied on TF plots by subtracting the prestimulus power between -250 and $-150 \mathrm{~ms}$ before stimulus onset, in each frequency band. To distinguish induced oscillatory activities from phase-locked evoked activities (i.e., transient and steady-state responses), we computed, at each point of the TF domain, the stimulus phase-locking factor (PLF) from the single-trial TF analysis (Tallon-Baudry et al., 1996). This factor ranges from 0 (uniform phase distribution, i.e., high latency jitter) to 1 (strict phase-locking to the stimulus). The Rayleigh statistic was used to test for the non-uniformity of phase distribution (Jervis et al., 1983). Therefore, with the number of trials used in this experiment, when the PLF was less than 0.17 , oscillations were considered to be non-phase-locked to the stimulus.

Statistical analysis. The statistical analysis in the two conditions, during both parts, focused on different electrophysiological components: the transient evoked responses to the onsets of parts 1 and 2, the slow wave responses elicited during the whole duration of the stimulus (sustained responses), the steady-state activities at 21,29 and $42 \mathrm{~Hz}$ (first harmonic of the $21 \mathrm{~Hz}$ ), and the induced gamma oscillations. As the data were not normally distributed, only non-parametric tests (Wilcoxon or Mann-Whitney) were used with a number of trials superior to 100 .

To identify, in each patient, the electrode contacts where a transient or sustained response was emerging, we computed a time varying Wilcoxon test from the single trials. It was applied on the mean amplitude of successive 20-ms windows between 0 and $200 \mathrm{~ms}$ and successive 100-ms windows between 200 and $800 \mathrm{~ms}$ after the onset of each part, compared to a prestimulus baseline (defined between -100 and $0 \mathrm{~ms}$ before part 1 onset). For contacts that showed a significant response, condition differences were estimated by a Mann-Whitney test applied to the same windows as described for the response identification test.

The emergence of steady-state activities was assessed by a Wilcoxon test on the mean power of each frequency $(21,29$ and $42 \mathrm{~Hz})$ over successive 100 -ms windows between 0 and $800 \mathrm{~ms}$ after the onset of each part, compared to the prestimulus baseline power (between -250 and $-150 \mathrm{~ms}$ before part 1 onset) of each frequency (note that the prestimulus baseline is shifted away from $0 \mathrm{~ms}$ because wavelet analysis tends to stretch out the early poststimulus low frequency components). For contacts that showed a significant steady-state response, condition differences were estimated by a Mann-Whitney test applied to the same windows as described for the response identification test. As the carrier frequencies of the $21-\mathrm{Hz}$ component were the same during parts 1 and 2 of the 2-stream condition and part 2 of the 1-stream condition, we compared, with a Mann-Whitney test, the mean power between 200 and $800 \mathrm{~ms}$ of part 2 for each condition to the mean power between 200 and $800 \mathrm{~ms}$ of part 1 in the 2-stream condition.

The emergence of oscillatory beta/gamma activities was detected in the time-frequency domain with a Wilcoxon test on the mean power of $100 \mathrm{~ms} \times 10 \mathrm{~Hz}$ windows (from 0 to $800 \mathrm{~ms}$ and from 20 to $100 \mathrm{~Hz}$ ) compared to the prestimulus power (between -250 and $-150 \mathrm{~ms}$ 
before part 1 onset) in the same frequency band. To circumscribe the components which are not phase-locked to the stimulus, i.e., induced oscillations, the combination of two criteria in the time-frequency domain was used: an emergence assessed by the Wilcoxon test and an absence of phase-locking to the stimulus assessed by the Rayleigh test $(\mathrm{PLF}<0.17)$, as defined above. The 1- and 2-stream conditions were then compared with a Mann-Whitney test on the mean power of $50 \mathrm{~ms} \times 6 \mathrm{~Hz}$ windows where induced gamma activities were previously found to emerge.

For each component, only those effects are discussed that met Bonferroni-corrected $p$-value criteria. In each patient, the probability threshold of 0.05 was thus divided by the number of tested windows over all investigated electrode contacts. The statistical analysis will mainly focus on the responses observed in part 2 during which the acoustical content of the stimuli were strictly the same but perception differed.

All signal analysis was performed with the ELAN-Pack software developed at INSERM U821.

Anatomical registration. Electrode contacts were $2 \mathrm{~mm}$ long and spaced every $3.5 \mathrm{~mm}$ (center-to-center). Depth probes (diameter $0.8 \mathrm{~mm}$ ) with 10 or 15 contacts each were inserted perpendicularly to the sagittal plane using Talairach's stereotactic grid (Talairach and Tournoux, 1988). Numbering of contacts is increasing from medial to lateral along an electrode track. Electrode locations were measured on X-ray images obtained in the stereotactic frame. The depth of penetration of each contact was measured on the frontal $X$-ray image from the tip of the electrode to the midline, which was visualized angiographically by the sagittal sinus. The co-registration of the lateral X-ray image and a midsagittal MRI scan, both having the same scale of 1 , allowed to measure the electrode coordinates in the individual Talaraich's space defined by the median sagittal plane, the AC-PC (anterior commissure - posterior commissure) horizontal plane and the vertical $A C$ frontal plane, these anatomical landmarks being identified on the 3D MRI scans. This procedure led to the superposition of each electrode contact onto the patients' structural MRIs. The accuracy of the registration procedure was $2 \mathrm{~mm}$, estimated on another patient's MR images obtained just after electrode explantation and in which electrode tracks were still visible.

Eventually, electrode contacts and experimental effects have been visualized on individual $3 \mathrm{D}$ rendering of isolated temporal cortices to facilitate the identification of auditory brain structures and the comparison of activated sites across subjects. The cortical surface was individually segmented by using FreeSurfer software (http://surfer.nmr. mgh.harvard.edu).

\section{Psychophysical experiment}

In a group of healthy subjects, a psychophysical experiment was used to ensure that the two types of stimuli implicitly induced two distinct perceptions. We considered that if perceptions during part 2 were different (whereas stimuli are acoustically identical during this part), behavioral performances in a stream-irrelevant auditory task could be differentially influenced. We chose to compare the detection thresholds of offset asynchrony for 1-stream and 2-stream stimuli.

Seventeen paid subjects participated in the behavioral study. All were free of neurological diseases and had normal hearing. Written informed consent was obtained from each subject.

A constant Yes/No procedure tracking 50\% correct responses was used to define the detection threshold of offset asynchrony. The 1-stream and 2-stream stimuli described above were randomly presented to the subjects. In this experiment, the $29-\mathrm{Hz}$ component could end either at the same time as the $21-\mathrm{Hz}$ component (as in the electrophysiological experiment) or slightly before (time difference: DT). Subjects had to indicate whether the two components were ending at the same time, or not, by pressing, with the same hand, a left or right button, respectively.

Only those subjects who have more than $65 \%$ of correct responses for both DT $=0 \mathrm{~ms}$ and their maximal DT value, were kept in the analysis. Only 10 subjects ( 6 men, age ranging from 22 to 34 years) fulfilled these criteria. Subject's psychometric function was fitted by a sigmoid function and the offset asynchrony detection threshold was defined as the DT value leading to $50 \%$ correct responses. The detection thresholds obtained in each condition were compared with a Wilcoxon test.

\section{RESULTS}

\section{Behavioral assessment of the stream perception induced in the two stimuli}

The aim of the psychophysical experiment was to ensure, by an indirect measure, that the two kinds of stimuli automatically induced two different perceptions (one stream or two streams) even when the subject is paying attention to aspects of the stimulus (e.g., offset asynchrony) that are not related to stream segregation. We chose to compare the detection thresholds of offset asynchrony for 1-stream and 2-stream stimuli. We found that the mean detection thresholds of offset asynchrony were significantly different in 1-stream and 2-stream conditions (52.1 and $71.5 \mathrm{~ms}$, respectively, $p=0.017$ ). Subjects were better at detecting offset asynchrony in the 1-stream rather than in the 2-stream condition.

In addition to the psychophysical experiment, we played the sounds to the subjects and asked them whether they heard one or two sounds during the second part. They always responded 'one' and 'two' for 1-stream and 2-stream conditions, respectively.

Moreover, four of the five patients also performed an active stream segregation task with the same 2-stream stimuli (Bidet-Caulet et al., 2007). They had to detect a spatial shift of the $21-\mathrm{Hz}$ component, which was impossible to perform without segregating the two streams. Their hit rate was ranging between 85 and $99 \%$ correct responses.

Thus we have shown, with these stimuli, that onset asynchrony explicitly induces stream segregation in patients and implicitly induces auditory segregation in normal subjects, as it has already been shown with other types of sounds (Bregman, 1990; Darwin et al., 1995; Turgeon et al., 2002; Turgeon et al., 2005). The electrophysiological experiment was performed on alert patients engaged in detecting stream-irrelevant sounds, and therefore, the observed brain responses could be related to implicit concurrent sound segregation/fusion.

\section{Anatomical data}

Three patients were implanted in the right hemisphere only, and two in both hemispheres. In all implanted hemispheres, at least one electrode track was located in the superior temporal plane. Electrodes $\mathrm{H}$ or $\mathrm{H}^{\prime}$ (for right or left hemisphere, respectively) were positioned posteriorly, passing through the Heschl's gyrus $(\mathrm{HG})$, the planum temporale $(\mathrm{PT})$ and the superior temporal gyrus (STG), and electrodes $\mathrm{T}, \mathrm{T}^{\prime}$ and $\mathrm{W}$ were positioned anteriorly, passing through the $\mathrm{HG}$ and the anterior PT or the planum polare (PP). Electrode $A, A^{\prime}, B, B^{\prime}$, and $C$ were penetrating through the middle temporal gyrus (MTG). Electrode $\mathrm{N}$ was located just above the superior temporal plane, in the parietal operculum.

Even though intracranial recordings in epileptic patients provide a sparse spatial sampling of the auditory cortex, we could access not only primary auditory areas (posteromedial part of the $\mathrm{HG}$ ) but also posterior and anterior secondary auditory regions.

\section{Electrophysiological components modulated as a function of perception}

Distinct electrophysiological components were identified in different parts of the auditory cortex. Figure 2 illustrates all these components, in the 1-stream condition, on the 3D rendering of patient \#4's temporal cortex. The 21, 29 and $42 \mathrm{~Hz}$ steady-state evoked activities were found in the posteromedial part of the $\mathrm{HG}$ and more anteriorly in the $\mathrm{HG}$. Transient and sustained evoked responses were observed at most of the electrode contacts located in the superior temporal plane: in the $\mathrm{HG}$, in the posterior part of the STG and in the planum temporale. Induced gamma oscillations, not phase-locked to stimulus onset, were found in the anterior part of the $\mathrm{HG}$ and in the lateral STG. 


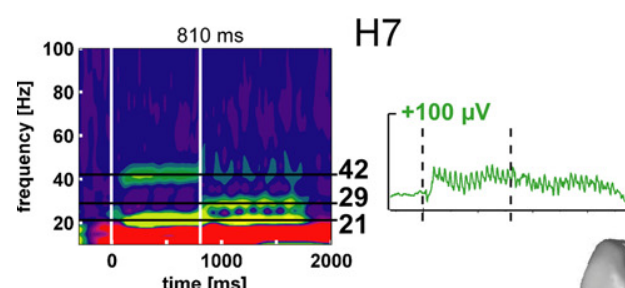

$\mathrm{H} 12$

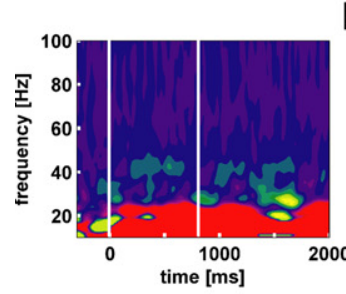

$\square_{-500}^{500}$ -

lanum temporale
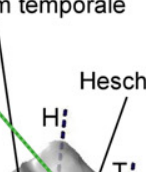

Heschl's gyrus

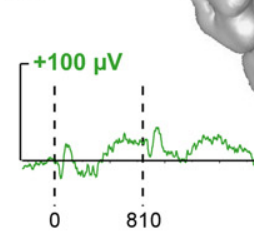

0

$1000 \quad 2000 \mathrm{~ms}$

- evoked responses

- induced activities

- phase locking factor
Time-frequency plots

T5

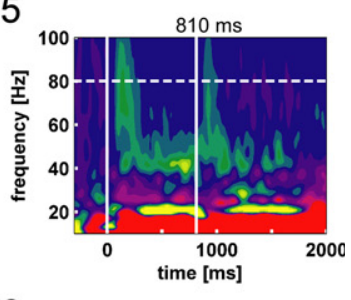

T6

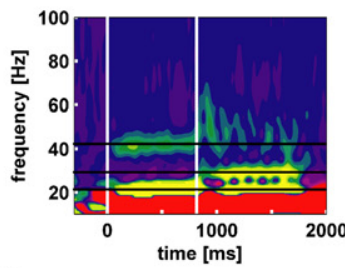

T9

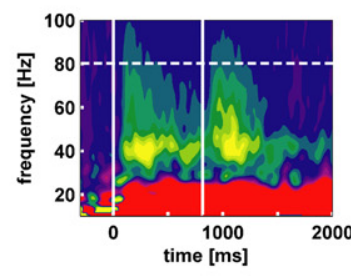

Figure 2. Illustration of the typical electrophysiological responses and their location in the 3D rendering of the temporal cortex (1-stream condition in patient \#4). Evoked responses (obtained by single-trial averaging) are plotted in green. Transient and sustained evoked responses were observed at most of the electrode contacts located in the superior temporal plane: in the Heschl's gyrus, HG (electrode contacts H7, T5 and T6), in the superior temporal gyrus, STG (T9) and in the planum temporale, PT (H12). The time-frequency plot of these activities (time-frequency power averaged after a wavelet-based analysis on each single trial) permits a good visualization of both evoked steady-state and gamma induced oscillations. Twenty-one hertz, 29 and $42 \mathrm{~Hz}$ steady-state evoked activities were found in the posteromedial part of the $\mathrm{HG}(\mathrm{H7})$ and more anteriorly in the $\mathrm{HG}$ (T6). Induced gamma oscillations were found in the anterior part of the HG (T5) and on the lateral STG (T9). The time profiles of induced activities at $80 \mathrm{~Hz}$ are depicted in red. When evoked and induced activities were present at the same place, in the same frequency band (see T5 as an example), the phase-locking factor indicated which activities were evoked (phase-locked to the stimulus: $P L F>0.17$ ). The time profiles of the phase-locking factor at $80 \mathrm{~Hz}$ are depicted in blue. All these responses are baseline corrected with respect to the prestimulus period preceding part 1 onset.

All these components were present during both parts 1 and 2 . Since part 1 is acoustically different in the 1 - and 2-stream conditions, we concentrated on the electrophysiological activities of part 2, during which, inputs were acoustically identical whereas perceptions, induced by the preceding part, were different. All 1- vs. 2-stream effects on the different electrophysiological components following part 2 onset will be presented, in all patients, on the 3D rendering of their individual temporal cortex. To illustrate the time-course of the responses, typical waveforms will be plotted from electrode contacts showing such effects.

\section{Transient and sustained evoked responses (4 patients)}

Most contacts of electrodes $\mathrm{H}, \mathrm{H}^{\prime}, \mathrm{T}, \mathrm{T}^{\prime}$ and $\mathrm{W}$ presented transient evoked responses. Three main transient waves were found after the onset of part 2. A first one was maximal between 50 and $80 \mathrm{~ms}$, a second one around $100 \mathrm{~ms}$ (between 80 and $150 \mathrm{~ms}$ ) and a third one starting around $150 \mathrm{~ms}$. The two first waves were significantly more prominent in the 1-stream condition, between 60 and $80 \mathrm{~ms}$ and between 80 and $150 \mathrm{~ms}$, on several contacts of the four patients kept for this analysis. The third one presented a steeper slope in the 1-stream condition, between 120 and $200 \mathrm{~ms}$. These effects were mainly located in the anterolateral $\mathrm{HG}$ and in the lateral part of the STG (Figure 3).

At most of the electrode contacts presenting transient responses, a sustained slow wave was present until the end of the stimulus, with generally no condition effect. Only in patient \#4, effects were found between
200 and $700 \mathrm{~ms}$ after the onset of part 2. In this case, the mean amplitude was significantly greater in the 2-stream condition. It should be noted that in the 2-stream condition very small transient evoked responses were generally elicited so that the sustained wave could begin earlier than in the 1-stream condition.

\section{Steady-state evoked activities}

Steady-state evoked responses were emerging at 21, 29 and $42 \mathrm{~Hz}$ on several contacts for all patients (Figure 4). Twenty-one hertz, 29 and $42 \mathrm{~Hz}$ activities could be observed at the same electrode contacts, but in most of the cases, their respective maximum values were at different adjacent contacts. A first focus was found bilaterally in the posteromedial part of the $\mathrm{HG}$ and a second one in the right hemisphere only, in a more anterior part of the $\mathrm{HG}$. These activities were emerging during several hundreds of $\mathrm{ms}$ and, in some cases, during the whole duration of part 2.

Twenty-one hertz and $42 \mathrm{~Hz}$ activities were found significantly greater in the 1-stream condition, in four and three patients, respectively. These effects were mainly concentrated between 400 and $700 \mathrm{~ms}$ for $21 \mathrm{~Hz}$, and between 300 and $700 \mathrm{~ms}$, for $42 \mathrm{~Hz}$ activities. No condition effect was found concerning the $29 \mathrm{~Hz}$ steady-state activity.

The evolution of the $21 \mathrm{~Hz}$ power over the whole stimulus duration was also analyzed. When comparing the $21 \mathrm{~Hz}$ power between part 2 of each condition and part 1 of the 2-stream condition (comparison of 200-800 ms time-windows after part 1 and 2 onset), we found that, 


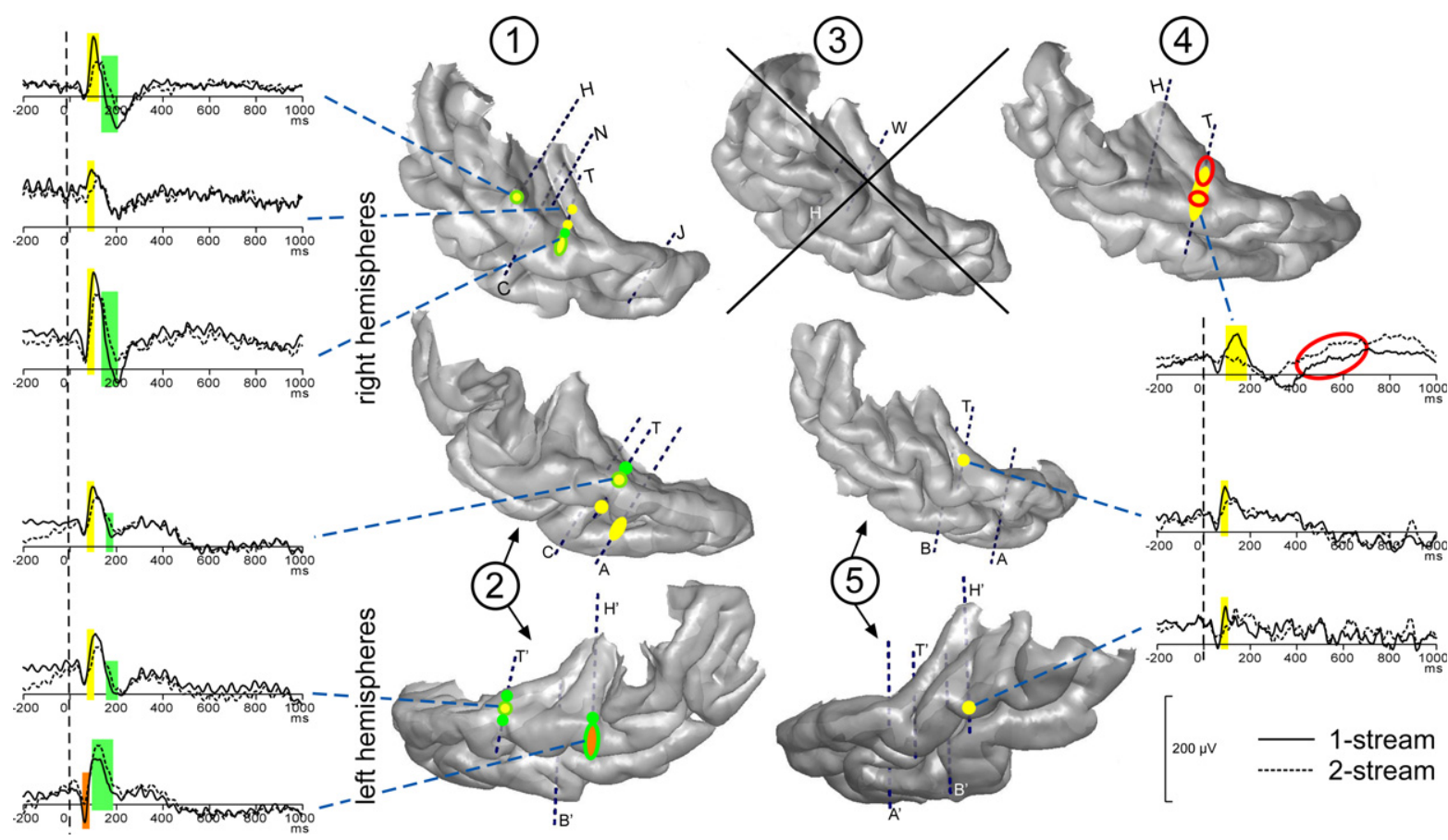

Figure 3. Modulations of evoked transient and sustained responses on the 3D rendering of individual temporal cortices. After the onset of part 2 ( $0 \mathrm{~ms})$, three main transient waves were found more prominent in the 1-stream condition, on several contacts of the four patients kept for this analysis. $A$ first one was modulated between 60 and $80 \mathrm{~ms}$ (orange), a second one between 80 and $160 \mathrm{~ms}$ (yellow) and a third one between 120 and $200 \mathrm{~ms}$ (green). These effects were mainly located on the lateral STG and their time-courses are depicted. In patient \#4, effects on the sustained response were found between 200 and 700 ms: mean amplitude was greater in the 2-stream condition (red circles). Yellow-green hatchings correspond to electrode contacts where both second and third effects are present. These evoked responses are baseline corrected with respect to the $(-100,0 \mathrm{~ms})$ period preceding part 1 . Each number corresponds to a patient, patients \#2 and 5 being implanted in both hemispheres.

(1)
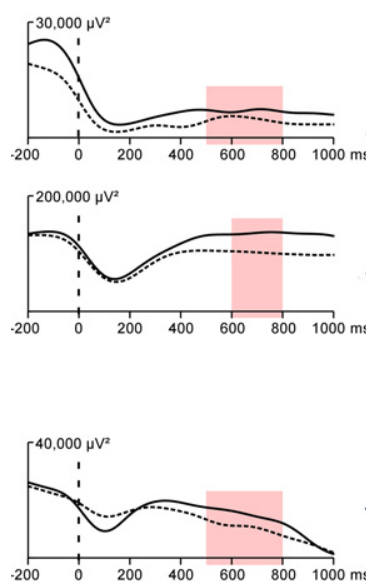

1-stream
.......... 2-stream
(3)

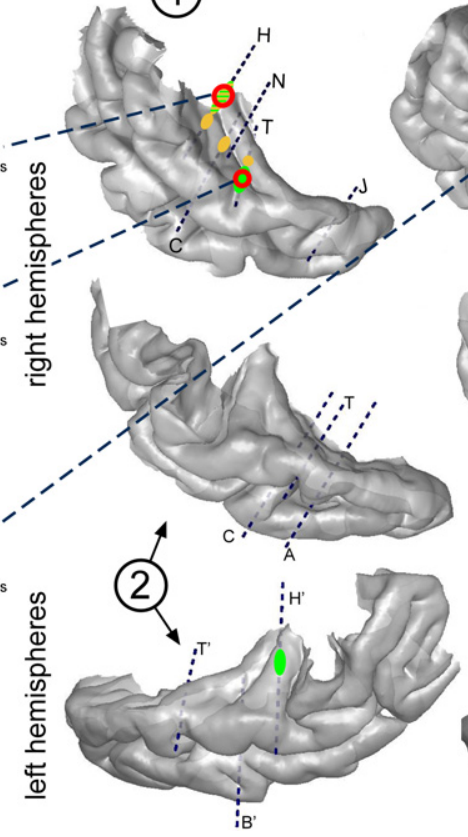

(4)

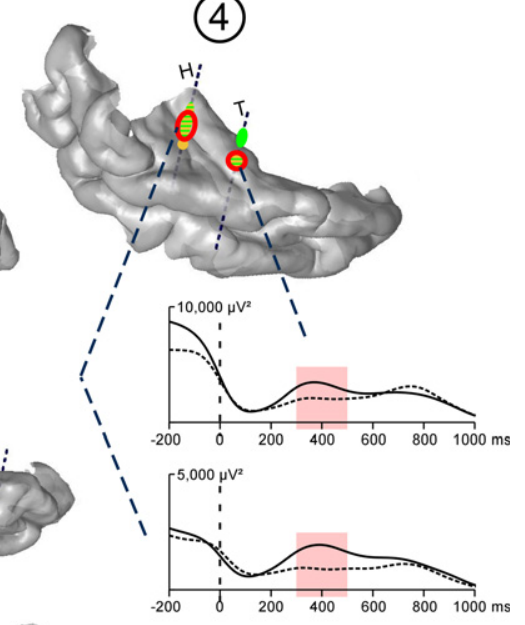

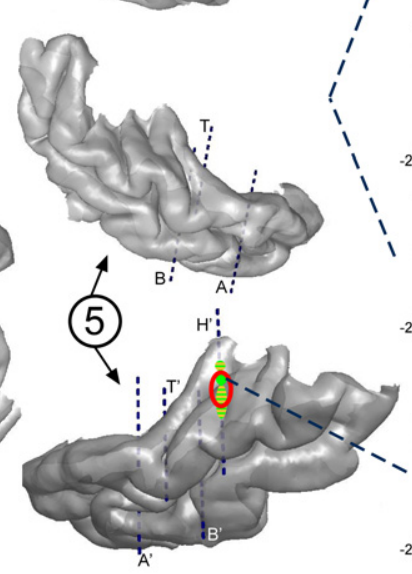

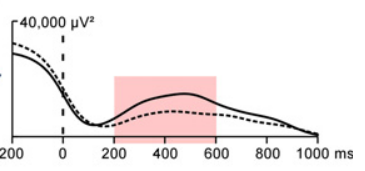

Figure 4. Emergence and modulations of evoked steady-state activities on the 3D rendering of individual temporal cortices. Emergence after the onset of part $2(0 \mathrm{~ms})$ are depicted on the temporal cortices, in green for 21 and yellow for $29 \mathrm{~Hz}$ steady-state activities. A first focus of steady-state activities was bilateral and located in the posterior part of the HG and a second one in the right hemisphere only, in a more anterior part of the HG. Twenty-one hertz activities were found to be enhanced in the 1-stream condition, in four patients ('1-stream > 2-stream', red circles). Time profiles at $21 \mathrm{~Hz}$ of these effects are plotted (significant differences are defined by light-red shaded areas). The power of these oscillatory activities is baseline corrected with respect to the (-250, $-150 \mathrm{~ms}$ ) period preceding part 1. Forty-two hertz steady-state emergence and effects presented quite similar topographies than $21 \mathrm{~Hz}$ steady-state activities. Each number corresponds to a patient, patients \#2 and 5 being implanted in both hemispheres. 


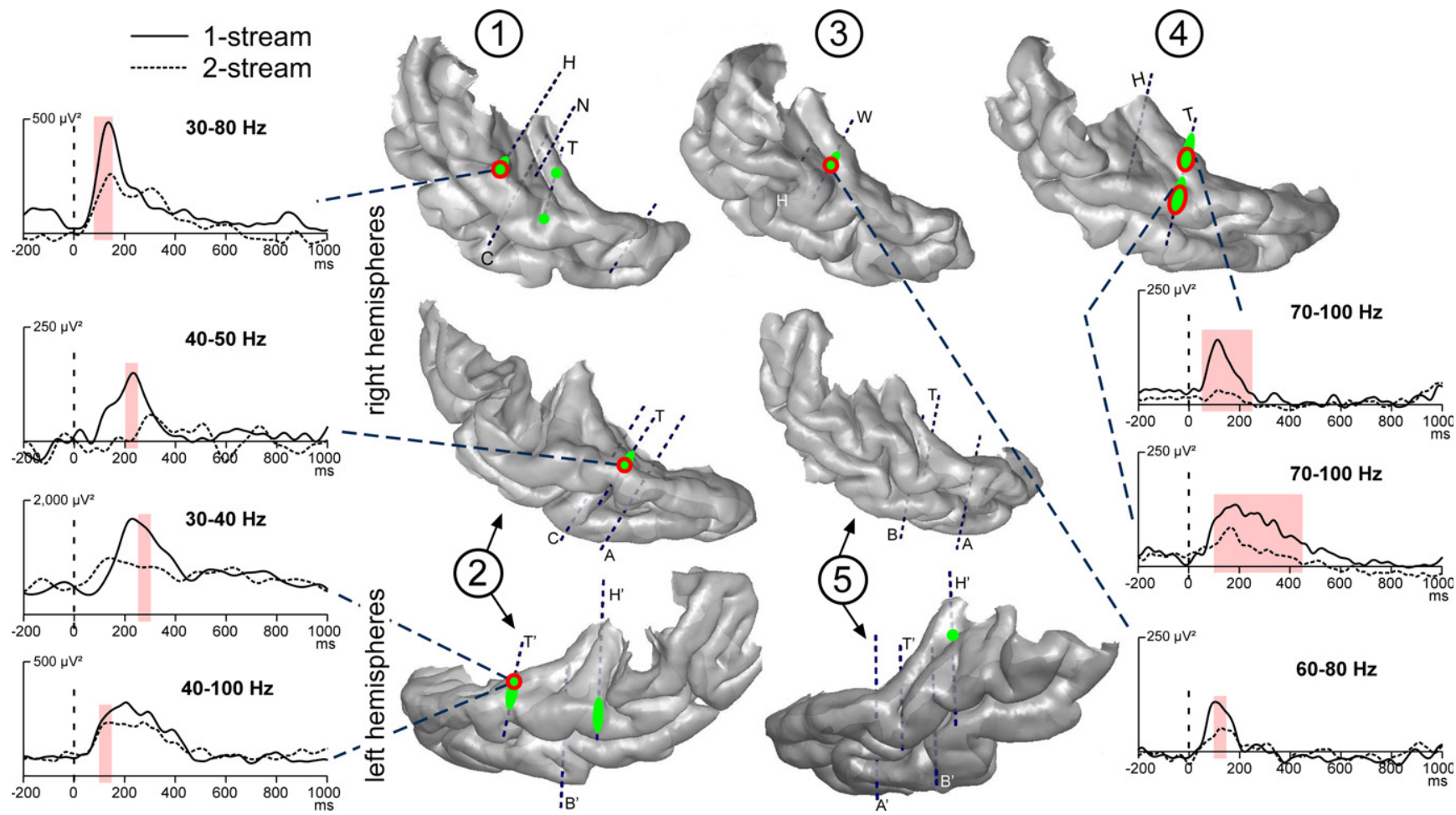

Figure 5. Emergence and modulations of induced gamma oscillations on the 3D rendering of individual temporal cortices. Emergence of induced gamma oscillations after the onset of part $2(0 \mathrm{~ms})$ is depicted in green. Induced gamma oscillations were emerging quite laterally in the HG and STG. They were found more prominent in the 1-stream condition, in four patients ("1-stream > 2-stream', red circles). At each electrode contact, significant effects were found in a specific frequency band. The gamma power time profiles of the corresponding frequency bands are plotted (significant differences are indicated by lightred shaded areas). The power of these oscillatory activities is baseline corrected with respect to the $(-250,-150$ ms) period preceding part 1 . Each number corresponds to a patient, patients \#2 and 5 being implanted in both hemispheres.

among the electrode contacts presenting a $21 \mathrm{~Hz}$ steady-state activity, 67 and $81 \%$ of them presented a greater activity during part 1 , and only 5 and $2 \%$ during part 2 , in the 1 -stream and 2 -stream conditions, respectively.

\section{Induced gamma oscillations}

Induced gamma oscillations were emerging at few contacts for each patient (Figure 5). They could be observed quite laterally in the $\mathrm{HG}$ and the STG. These activities were most frequently emerging between 100 and $350 \mathrm{~ms}$ in the $50-90 \mathrm{~Hz}$ frequency band (Figure 6). They were found significantly more prominent in the 1-stream condition, in four patients. These effects were quite distributed in the time-frequency (TF) domain (peaking between 150 and $300 \mathrm{~ms}$ ) and were lasting $100 \mathrm{~ms}$ on average.

\section{Topographies of the different electrophysiological activities}

The effects found for each component were combined on the individual 3D cortical renderings (Figure 7) to compare their topographies. Effects on induced gamma oscillations and transient evoked responses were mainly found in the lateral STG with a tendency to overlap. Effects on steady-state activities were more medial in the $\mathrm{HG}$ and had little overlap with other activities.

\section{DISCUSSION}

In the present experiment, we manipulated the onsets of two concurrent sounds to generate different percepts (segregation or fusion). We found that transient and steady-state evoked responses, and induced gamma oscillations, directly recorded in the human superior temporal cortex, are more pronounced in the case of onset synchrony of the two concurrent sounds than in the case of onset asynchrony. These findings

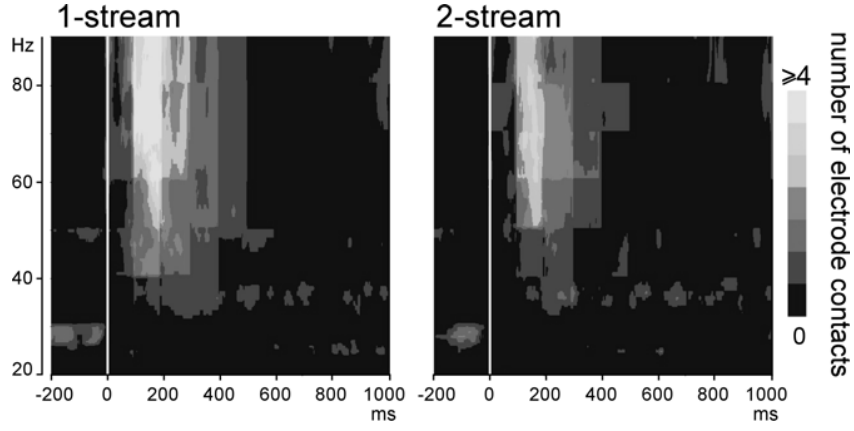

Figure 6. Emergence of induced gamma oscillations after the onset of part $2(0 \mathrm{~ms})$. Representation, in the time-frequency domain, of the mean number of electrode contacts across patients which presented significantly emerging induced gamma oscillations. By means of a time-frequency criteria based on the stimulus phase-locking factor (PLF), only non-phaselocked induced activities are represented here. These activities were most frequently present between 100 and $350 \mathrm{~ms}$ in the $50-90 \mathrm{~Hz}$ frequency band.

are noteworthy, because they provide both a precise time-course and a detailed localization of the neurophysiological correlates of concurrent sound perception in humans. Indeed, investigating epileptic patients with intracranial recordings represents a rare opportunity to combine high spatio-temporal resolution of brain activities with well controlled behavioral measures. The earliest effect was found around $60 \mathrm{~ms}$ on the transient evoked responses elicited in secondary auditory areas (posterior lateral STG). This effect is subsequently spreading over the PT and the 


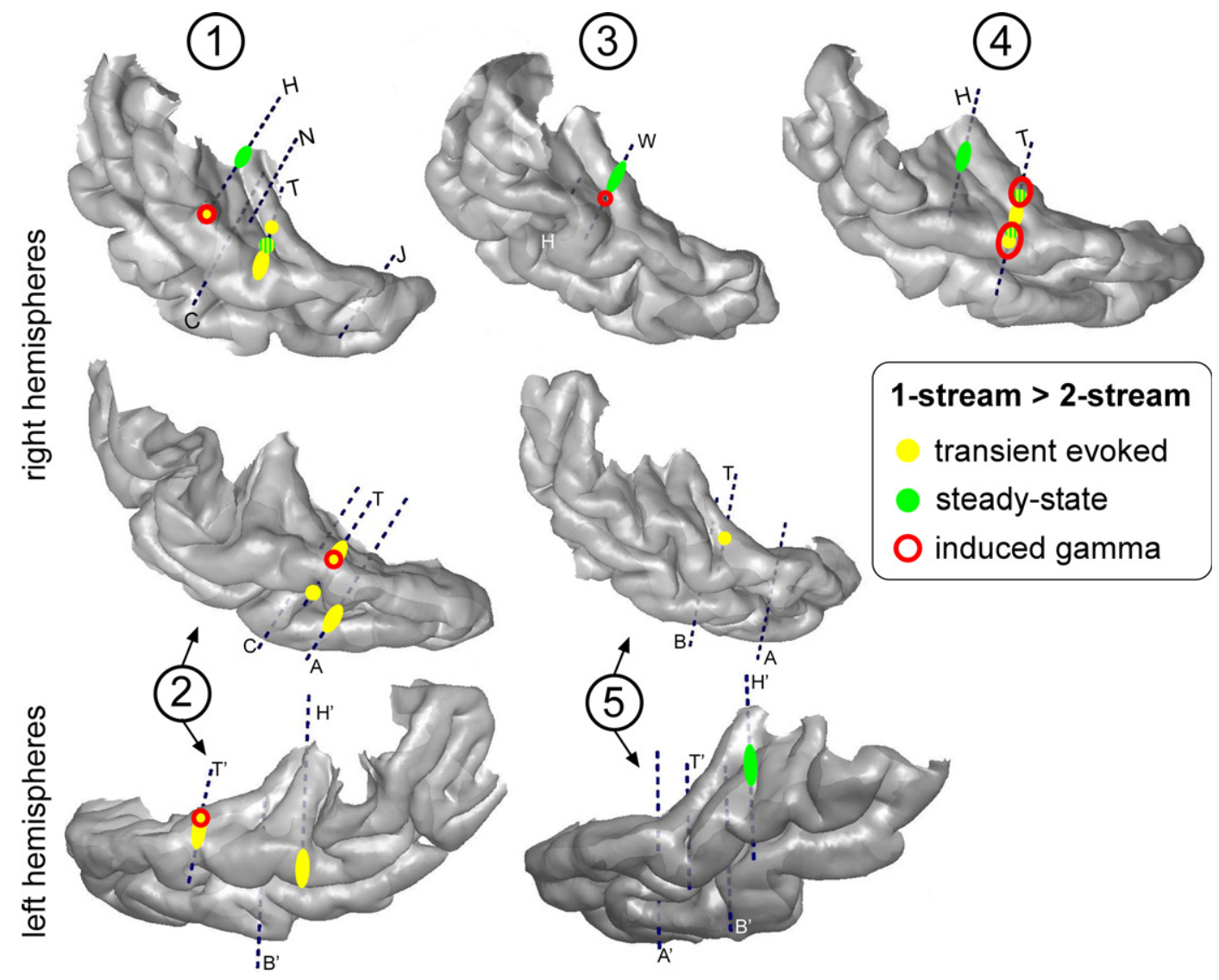

Figure 7. Topographies of the different electrophysiological activities on the 3D rendering of individual temporal cortices. The effects ' 1 -stream $>2$-stream' after the onset of part 2 are depicted in yellow for transient evoked responses, green for steady-state activities and red for induced gamma oscillations. Effects for induced gamma oscillations and transient evoked responses were mainly found in the lateral STG with a tendency to overlap. Effects for steady-state activities were more medial in the $\mathrm{HG}$ and had little overlap with other activities. Each number corresponds to a patient, patients \#2 and 5 being implanted in both hemispheres.

lateral STG, until $200 \mathrm{~ms}$. Induced gamma oscillations were then modulated in the same or nearby regions, until $300 \mathrm{~ms}$. Steady-state evoked responses were in turn affected during several hundreds of $\mathrm{ms}$ in the primary auditory cortex and in the anterolateral part of the $\mathrm{HG}$, in the right hemisphere.

\section{Spatio-temporal characterization of the auditory responses}

Three transient evoked responses were found significantly enhanced in the 1-stream condition. The first wave, between 50 and $80 \mathrm{~ms}$, was more pronounced in the lateral $\mathrm{HG}$, in one patient only. The two later waves around 100 and 150-200 ms were more pronounced at two foci, one in the most anterior and lateral part of the PT and one more posteriorly. These effects are thus mainly located in associative areas, and not in the primary auditory cortex (PAC), located in the posteromedial part of the $\mathrm{HG}$ (Liegeois-Chauvel et al., 1991; Rivier and Clarke, 1997). The topographical distribution of those waves is similar to that found for tone bursts in previous intracranial studies (Howard et al., 2000; Liegeois-Chauvel et al., 1994).

The 21,29 and $42 \mathrm{~Hz}$ steady-state activities were found bilaterally in the posteromedial part of the $\mathrm{HG}$, corresponding to the PAC, and in the right hemisphere only, in the anterolateral part of the $\mathrm{HG}$, considered as a secondary auditory area. The origin of steady-state activities in the PAC is consistent with previous studies (Gutschalk et al., 1999; Liegeois-Chauvel et al., 2004). Moreover, Gutschalk et al. (1999) found an additional generator of $40 \mathrm{~Hz}$ steady-state activity in the lateral HG, bilaterally, and Liegeois-Chauvel et al. (2004) in different auditory associative areas according to the frequency of the amplitude modulation.

Induced gamma oscillations were emerging, in the lateral $\mathrm{HG}$ and STG, in the $50-90 \mathrm{~Hz}$ frequency band, between 100 and $350 \mathrm{~ms}$ after the onset of part 2. With electrocorticographic (ECoG) recordings, similar activities were found in primary and secondary auditory areas in rats (Franowicz and Barth, 1995) and anesthetized monkeys (Brosch et al., 2002), and in the human STG (Crone et al., 2001; Edwards et al., 2005). The time-frequency characteristics of the observed induced gamma are consistent with previous MEG findings (Pantev, 1995). To our knowledge, the present study constitutes the first attempt to precisely localize these gamma oscillations in the human superior temporal plane, since ECoG does not permit to disentangle the contribution of different auditory regions ( $\mathrm{HG}, \mathrm{PT}, \mathrm{PP})$. Our results show that induced gamma oscillations can be elicited in several non-primary auditory areas.

\section{Role of the frequency selectivity of auditory areas}

In the present study, we manipulated the acoustical context during the part 1 (sound onset asynchrony/synchrony) to induce the segregation/ fusion of the two concurrent sounds during the part 2. The two kinds of stimuli were thus acoustically different during the part 1 only. For the 2 -stream stimuli, the carrier frequency of the $21-\mathrm{Hz}$ component was maintained throughout parts 1 and 2, whereas, for 1-stream stimuli, it presents a discontinuity at part 2 onset. Therefore, the only acoustic difference between the two conditions was the pitch continuity or discontinuity of the $21-\mathrm{Hz}$ component between parts 1 and 2 . 
As several human auditory areas, including the lateral $\mathrm{HG}$ and the PT, are known to be tonotopically organized (Formisano et al., 2003; Howard et al., 1996; Pantev et al., 1995; Romani et al., 1982; Talavage et al., 2004), a pitch discontinuity most likely activates new neural populations contributing to additional electrophysiological components. Thus, in the 1-stream condition, transient evoked response enhancement can be due to an additional $0 \mathrm{~N}$-response induced by the pitch discontinuity. If an OFF-response to the offset of the $21-\mathrm{Hz}$ component of part $1 \mathrm{can}$ not be ruled out, its contribution is probably minor (Makela et al., 1988). Even though this effect is found in the lateral STG, a contribution, through parallel thalamocortical pathways (Kaas et al., 1999), of subcortical relay nuclei, also known to be tonotopically organized, may also be considered. Because of relatively limited signal-to-noise ratio, we can not rule out the existence of an effect on earlier components in the PAC.

Similarly to the transient evoked responses, the enhancement of induced gamma oscillations in the 1-stream condition could be due to the frequency selectivity of neurons in the lateral STG. However, if, in monkey, the occurrence of induced gamma oscillations has been found to be related to frequency-selective neurons in the primary auditory area and in the caudomedial field, probably homologous of the human medial PT (Brosch et al., 2002), no such frequency selectivity of induced oscillations has been reported in the lateral STG.

Therefore, the enhancement of transient evoked responses and induced gamma oscillations, in the 1-stream condition, could be related to the frequency selectivity of the tonotopically organized auditory cortical fields. Transient evoked responses and induced gamma oscillations could reflect the encoding of low-level information, like sound changes (e.g., sound onset and pitch step), and also the simultaneity of these sound changes.

\section{The perceptual binding hypothesis in the context of concurrent sound perception}

An alternative interpretation of the induced gamma oscillation enhancement could be based on the 'perceptual binding hypothesis' (von der Malsburg and Schneider, 1986). This hypothesis proposes that induced gamma oscillations could correspond to the neural mechanism by which the different areas, involved in the processing of distinct components of the same object, could interact together to lead to the construction of a coherent object (Tallon-Baudry and Bertrand, 1999). Our results are consistent with this hypothesis, since induced gamma oscillations are more pronounced when the two sounds are grouped together into one complex stream.

This grouping process seems to involve high-frequency gamma oscillations (i.e., above $50 \mathrm{~Hz}$ ). This observation is consistent with recent MEG findings in the visual modality (Vidal et al., 2006) showing that induced high gamma oscillations can be modulated by the grouping properties of visual stimuli, high gamma being larger in a one-group condition than in two-group conditions irrespective of attention orientation. Therefore, as in the visual system, high-frequency gamma oscillations can be automatically induced in the auditory cortex by an acoustic event, and seem to be modulated in amplitude according to the number of perceptual groups formed, even in case of diverted attention.

\section{Habituation of steady-state responses}

The 21 and $42 \mathrm{~Hz}$ steady-state activities were found significantly greater in the 1-stream than in the 2-stream condition, between 300 and $700 \mathrm{~ms}$ during part 2. In fact, this effect does not correspond to an enhancement of those activities in the 1-stream condition, but rather to a greater reduction in the 2-stream condition. Indeed, it appeared that the power of steady-state activities tends to decrease with repetition, as it is observed from the onset to the end of the stimulus in both conditions. This diminution is most likely to be related to habituation mechanisms (Thompson and Spencer, 1966). The reason why these activities were found less reduced in the 1-stream condition (whereas the acoustic inputs were identical) is certainly the change in pitch of the $21-\mathrm{Hz}$ component. Since steady-state activities have been shown to be generated in the PAC and the anterolateral part of the $\mathrm{HG}$, known as tonotopically organized regions (Pantev et al., 1996; Romani et al., 1982), they should be sensitive to pitch changes and to frequency-selective habituation mechanisms. Thus, in the 1-stream condition, an habituation process developed during part 1, leading to a progressive reduction of the $21 \mathrm{~Hz}$ activity. This decrease was interrupted by the pitch discontinuity, which elicited an enhanced $21 \mathrm{~Hz}$ activity, selective of the new pitch of the $21-\mathrm{Hz}$ component. In the 2-stream condition, the habituation process also developed and produced a diminution of the continuous $21 \mathrm{~Hz}$ activity, progressively until the end of the stimulus. Thus the $21 \mathrm{~Hz}$ response is more reduced in the 2-stream than in the 1-stream condition whereas the $29-\mathrm{Hz}$ response is similar.

Therefore, effects on steady-state responses can be explained by the combination of two mechanisms, frequency selectivity and habituation of the auditory cortical fields. By modulating the weight of the $21-\mathrm{Hz}$ response, these mechanisms lead to different amplitude ratios between 29 and $21 \mathrm{~Hz}$ activities in the two conditions. This ratio is greater in the 2 -stream than in the 1-stream condition. In the case of pitch continuity (onset asynchrony), the $21-\mathrm{Hz}$ response being highly reduced, the $29-\mathrm{Hz}$ response becomes relatively more important. This could contribute to the increased saliency of the new-coming $29-\mathrm{Hz}$ component, leading to the segregation into two streams. Conversely, in the case of pitch discontinuity (onset synchrony), the $21-\mathrm{Hz}$ response being weakly reduced, the $29-\mathrm{Hz}$ component tends to merge into the acoustic mixture and the two components are grouped into one complex stream.

\section{Relationship with electrophysiological studies of stream segregation}

Many studies have explored sequential stream segregation, but very few have investigated the neural mechanisms involved in the perception of sounds that overlap in time. With the exception of binaural cues, studies of concurrent sound segregation have focused on the mistuning of a component from an harmonic complex. Our results are partly consistent with previous human findings (Alain et al., 2001, 2002; Dyson and Alain, 2004) showing that different electrophysiological waves originating in the auditory cortex (between 30 and $200 \mathrm{~ms}$ ) were modulated according to the stimulus type, tuned or mistuned. The authors further proposed that their peak enhancement at $180 \mathrm{~ms}$ in case of mistuned stimuli could be due to an additional component, the 'object-related-negativity', associated with the segregation of simultaneous auditory objects. This effect could also be explained, as in the present experiment, by low-level processing of the acoustic contents which differed between tuned and mistuned stimuli.

In the present experiment, the electrophysiological effects can be directly related to acoustic differences between the two conditions (onset asynchrony or synchrony, i.e., pitch continuity or discontinuity), probably involving frequency selectivity and habituation mechanisms. In this concurrent sound context, the manipulation of onset synchrony induces the perception of one or two streams. The observed differences in the electrophysiological responses (due to acoustic differences between the two conditions) could thus participate in the construction of one or two stream percepts.

Therefore, the present study suggests that frequency selectivity and habituation mechanisms could be involved in the grouping or segregation of concurrent sounds. This is highly consistent with recent findings in the macaque PAC during sequential stream segregation (Micheyl et al., 2005). It has also been reported that sequential stream segregation could be based on low-level mechanisms, such as frequency selectivity and selective adaptation or suppression, in songbirds (Bee and Klump, 2004), in mustached bats (Kanwal et al., 2003), in monkeys (Fishman et al., 2001, 2004) and in humans (Gutschalk et al., 2005; Snyder et al., 2006).

\section{CONCLUSION}

From the present neurophysiological observations, a possible interpretation of the dynamics of the neural interactions subtending concurrent sound perception could be proposed. Firstly, as can be observed on transient evoked responses, basic acoustic features are encoded in 
frequency-selective regions, without excluding a possible participation of sub-cortical relays to this selective encoding. Secondly, at a cortical level gamma oscillations could mediate the binding of these active regions for the construction of a single coherent auditory stream. Lastly, steadystate activities in the primary auditory cortex, modulated by habituation mechanisms, could contribute to the maintenance of the 1- or 2-stream percept.

We could not definitely conclude whether the observed electrophysiological differences are related to the perception of two streams or to acoustic changes. However, we describe the neurophysiological correlates of the impact of the acoustic context on concurrent sound perception, specifically in terms of frequency selectivity and habituation mechanisms. The remaining questions are where and when in the brain these neural patterns could be integrated to build up stream percept. This study suggests that further investigations of gamma oscillations in auditory grouping could bring new insights on this phenomenon.

\section{CONFLICT OF INTEREST STATEMENT}

The authors declare that the research was conducted in the absence of any commercial or financial relationships that could be construed as a potential conflict of interest.

\section{ACKNOWLEDGEMENT}

We thank Nicolas Grimault (CNRS-UMR5020, Lyon) for helpful discussions

\section{REFERENCES}

Alain, C., Arnott, S. R., and Picton, T. W. (2001). Bottom-up and top-down influences on auditory scene analysis: evidence from event-related brain potentials. J. Exp. Psychol. Hum. Percept. Perform. 27, 1072-1089.

Alain, C., and Izenberg, A. (2003). Effects of attentional load on auditory scene analysis J. Cogn. Neurosci. 15, 1063-1073.

Alain, C., Schuler, B. M., and McDonald, K. L. (2002). Neural activity associated with distinguishing concurrent auditory objects. J. Acoust. Soc. Am. 111, 990-995.

Bee, M. A., and Klump, G. M. (2004). Primitive auditory stream segregation: a neurophysiological study in the songbird forebrain. J. Neurophysiol. 92, 1088-1104.

Bertrand, 0., and Tallon-Baudry, C. (2000). Oscillatory gamma activity in humans: a possible role for object representation. Int. J. Psychophysiol. 38, 211-223.

Bidet-Caulet, A., Fischer, C., Besle, J., Aguera, P. E., Giard, M. H., and Bertrand, 0. (2007) Effects of selective attention on the electrophysiological representation of concurrent sounds in the human auditory cortex. J. Neurosci. 27, 9252-9261.

Botte, M. C., Canévet, G., Demany, L., and Sorin, C. (1989). Psychoacoustique et Perception Auditive. Paris, INSERM/SFA/CNET.

Bregman, A. (1990). Auditory Scene Analysis: The Perceptual Organization of Sound. Cambridge, MA, MIT Press.

Brosch, M., Budinger, E., and Scheich, H. (2002). Stimulus-related gamma oscillations in primate auditory cortex. J. Neurophysiol. 87, 2715-2725.

Crone, N. E., Boatman, D., Gordon, B., and Hao, L. (2001). Induced electrocorticographic gamma activity during auditory perception. Clin. Neurophysiol. 112, 565-582.

Darwin, C. J., Hukin, R. W., and al-Khatib, B. Y. (1995). Grouping in pitch perception: evidence for sequential constraints. J. Acoust. Soc. Am. 98, 880-885.

Draganova, R., Ross, Borgmann, B., and Pantev, C. (2002). Auditory cortical response patterns to multiple rhythms of AM sound. Ear Hear. 23, 254-265.

Dyson, B. J., and Alain, C. (2004). Representation of concurrent acoustic objects in primary auditory cortex. J. Acoust. Soc. Am. 115, 280-288.

Edwards, E., Soltani, M., Deouell, L. Y., Berger, M. S., and Knight, R. T. (2005). High gamma activity in response to deviant auditory stimuli recorded directly from human cortex. J. Neurophysiol. 94, 4269-4280.

Fishman, Y. I., Arezzo, J. C., and Steinschneider, M. (2004). Auditory stream segregation in monkey auditory cortex: effects of frequency separation, presentation rate, and tone duration. J. Acoust. Soc. Am. 116, 1656-1670.

Fishman, Y. I., Reser, D. H., Arezzo, J. C., and Steinschneider, M. (2001). Neural correlates of auditory stream segregation in primary auditory cortex of the awake monkey. Hear. Res. 151, 167-187.

Formisano, E., Kim, D. S., Di Salle, F., van de Moortele, P. F., Ugurbil, K., and Goebel, R. (2003). Mirror-symmetric tonotopic maps in human primary auditory cortex. Neuron 40, 859-869.

Franowicz, M. N., and Barth, D. S. (1995). Comparison of evoked potentials and highfrequency (gamma-band) oscillating potentials in rat auditory cortex. J. Neurophysiol. 74, 96-112.

Gutschalk, A., Mase, R., Roth, R., Ille, N., Rupp, A., Hahnel, S. et al. (1999). Deconvolution of $40 \mathrm{~Hz}$ steady-state fields reveals two overlapping source activities of the human auditory cortex. Clin. Neurophysiol. 110, 856-868.
Gutschalk, A., Micheyl, C., Melcher, J. R., Rupp, A., Scherg, M., and Oxenham, A. J. (2005). Neuromagnetic correlates of streaming in human auditory cortex. J. Neurosci. 25, 5382-5388

Howard, M. A. III, Volkov, I. O., Abbas, P. J., Damasio, H., Ollendieck, M. C., and Granner, M. A. (1996). A chronic microelectrode investigation of the tonotopic organization of human auditory cortex. Brain Res. 724, 260-264.

Howard, M. A., Volkov, I. O., Mirsky, R., Garell, P. C., Noh, M. D., Granner, M. et al. (2000). Auditory cortex on the human posterior superior temporal gyrus. J. Comp. Neurol. 416, 79-92.

Jervis, B. W., Nichols, M. J., Johnson, T. E., Allen, E., and Hudson, N. R. (1983). A fundamental investigation of the composition of auditory evoked potentials. IEEE Trans. Biomed. Eng. 30, 43-50.

Kaas, J. H., Hackett, T. A., and Tramo, M. J. (1999). Auditory processing in primate cerebral cortex. Curr. Opin. Neurobiol. 9, 164-170.

Kanwal, J. S., Medvedev, A. V., and Micheyl, C. (2003). Neurodynamics for auditory stream segregation: tracking sounds in the mustached bat's natural environment. Network 14, 413-435.

Liegeois-Chauvel, C., Lorenzi, C., Trebuchon, A., Regis, J., and Chauvel, P. (2004). Temporal envelope processing in the human left and right auditory cortices. Cereb. Cortex 14, 731-740.

Liegeois-Chauvel, C., Musolino, A., Badier, J. M., Marquis, P., and Chauvel, P. (1994). Evoked potentials recorded from the auditory cortex in man: evaluation and topography of the middle latency components. Electroencephalogr. Clin. Neurophysiol. 92, 204-214.

Liegeois-Chauvel, C., Musolino, A., and Chauvel, P. (1991). Localization of the primary auditory area in man. Brain 114, 139-151.

Lins, 0. G., and Picton, T. W. (1995). Auditory steady-state responses to multiple simultaneous stimuli. Electroencephalogr. Clin. Neurophysiol. 96, 420-432.

Makela, J. P., Hari, R., and Leinonen, L. (1988). Magnetic responses of the human auditory cortex to noise/square wave transitions. Electroencephalogr. Clin. Neurophysiol. $69,423-430$.

Micheyl, C., Carlyon, R. P., Gutschalk, A., Melcher, J. R., Oxenham, A. J., Rauschecker, J. P. et al. (2007). The role of auditory cortex in the formation of auditory streams. Hear. Res. 229, 116-131.

Micheyl, C., Tian, B., Carlyon, R. P., and Rauschecker, J. P. (2005). Perceptual organization of tone sequences in the auditory cortex of awake macaques. Neuron 48, 139-148.

Nager, W., Kohlmetz, C., Joppich, G., Mobes, J., and Munte, T. F. (2003). Tracking of multiple sound sources defined by interaural time differences: brain potential evidence in humans. Neurosci. Lett. 344, 181-184.

Pantev, C. (1995). Evoked and induced gamma-band activity of the human cortex. Brain Topogr. 7, 321-330.

Pantev, C., Bertrand, O., Eulitz, C., Verkindt, C., Hampson, S., Schuierer, G. et al. (1995). Specific tonotopic organizations of different areas of the human auditory cortex revealed by simultaneous magnetic and electric recordings. Electroencephalogr. Clin. Neurophysiol. 94, 26-40.

Pantev, C., Roberts, L. E., Elbert, T., Ross, B., and Wienbruch, C. (1996). Tonotopic organization of the sources of human auditory steady-state responses. Hear. Res. 101, 62-74.

Rivier, F., and Clarke, S. (1997). Cytochrome oxidase, acetylcholinesterase, and NADPHdiaphorase staining in human supratemporal and insular cortex: evidence for multiple auditory areas. Neuroimage 6, 288-304.

Romani, G. L., Williamson, S. J., and Kaufman, L. (1982). Tonotopic organization of the human auditory cortex. Science 216, 1339-1340.

Sinex, D. G., Sabes, J. H., and Li, H. (2002). Responses of inferior colliculus neurons to harmonic and mistuned complex tones. Hear. Res. 168, 150-162.

Snyder, J. S., Alain, C., and Picton, T. W. (2006). Effects of attention on neuroelectric correlates of auditory stream segregation. J. Cogn. Neurosci. 18, 1-13.

Sussman, E. S., Bregman, A. S., Wang, W. J., and Khan, F. J. (2005). Attentional modulation of electrophysiological activity in auditory cortex for unattended sounds within multistream auditory environments. Cogn. Affect. Behav. Neurosci. 5, 93-110.

Sussman, E., Ritter, W., and Vaughan, H. G. Jr (1999). An investigation of the auditory streaming effect using event-related brain potentials. Psychophysiology 36, 22-34.

Takegata, R., Brattico, E., Tervaniemi, M., Varyagina, 0., Naatanen, R., and Winkler, I. (2005). Preattentive representation of feature conjunctions for concurrent spatially distributed auditory objects. Brain Res. Cogn. Brain Res. 25, 169-179.

Talairach, J., and Tournoux, P. (1988). Co-Planar Stereotaxic Atlas of the Human Brain. New York, NY, Thieme Medical Publishers.

Talavage, T. M., Sereno, M. I., Melcher, J. R., Ledden, P. J., Rosen, B. R., and Dale, A. M. (2004). Tonotopic organization in human auditory cortex revealed by progressions of frequency sensitivity. J. Neurophysiol. 91, 1282-1296.

Tallon-Baudry, C., and Bertrand, 0. (1999). Oscillatory gamma activity in humans and its role in object representation. Trends Cogn. Sci. 3, 151-162.

Tallon-Baudry, C., Bertrand, 0., Delpuech, C., and Pernier, J. (1996). Stimulus specificity of phase-locked and non-phase-locked $40 \mathrm{~Hz}$ visual responses in human. J. Neurosci. 16, 4240-4249.

Thompson, R. F., and Spencer, W. A. (1966). Habituation: a model phenomenon for the study of neuronal substrates of behavior. Psychol. Rev. 73, 16-43. 
Turgeon, M., Bregman, A. S., and Ahad, P. A. (2002). Rhythmic masking release: contribution of cues for perceptual organization to the cross-spectral fusion of concurrent narrow-band noises. J. Acoust. Soc. Am. 111, 1819-1831.

Turgeon, M., Bregman, A. S., and Roberts, B. (2005). Rhythmic masking release: effects of asynchrony, temporal overlap, harmonic relations, and source separation on cross-spectral grouping. J. Exp. Psychol. Hum. Percept. Perform. 31, 939-953.
Vidal, J. R., Chaumon, M., O'Regan, J. K., and Tallon-Baudry, C. (2006). Visual grouping and the focusing of attention induce gamma-band oscillations at different frequencies in human magnetoencephalogram signals. J. Cogn. Neurosci. 18, 1850-1862. von der Malsburg, C., and Schneider, W. (1986). A neural cocktail-party processor. Biol. Cybern. 54, 29-40.

Winkler, I., Teder-Salejarvi, W. A., Horvath, J., Naatanen, R., and Sussman, E. (2003). Human auditory cortex tracks task-irrelevant sound sources. Neuroreport 14, 2053-2056. 